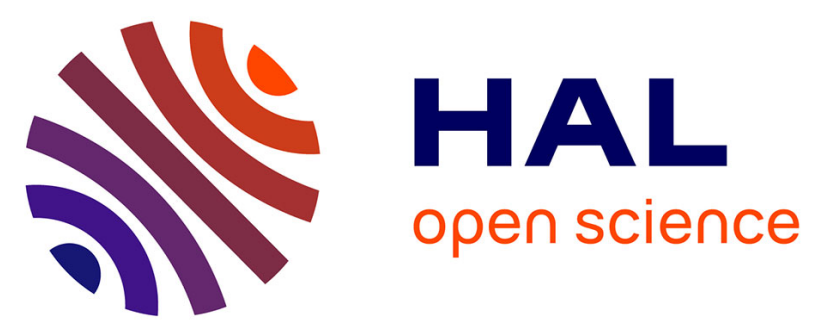

\title{
Analysis of anatomical variability using diffeomorphic iterative centroid in patients with Alzheimer's disease
}

Claire Cury, Joan Glaunès, Marie Chupin, Olivier Colliot

\section{To cite this version:}

Claire Cury, Joan Glaunès, Marie Chupin, Olivier Colliot. Analysis of anatomical variability using diffeomorphic iterative centroid in patients with Alzheimer's disease. Computer Methods in Biomechanics and Biomedical Engineering: Imaging \& Visualization, 2017, 5 (5), pp.350 - 358. 10.1080/21681163.2015.1035403 . hal-01135304

\section{HAL Id: hal-01135304 \\ https://inria.hal.science/hal-01135304}

Submitted on 6 Jan 2016

HAL is a multi-disciplinary open access archive for the deposit and dissemination of scientific research documents, whether they are published or not. The documents may come from teaching and research institutions in France or abroad, or from public or private research centers.
L'archive ouverte pluridisciplinaire HAL, est destinée au dépôt et à la diffusion de documents scientifiques de niveau recherche, publiés ou non, émanant des établissements d'enseignement et de recherche français ou étrangers, des laboratoires publics ou privés. 
To appear in Computer Methods in Biomechanics and Biomedical Engineering

Vol. 00, No. 00, Month 20XX, 1-10

\title{
Analysis of anatomical variability using diffeomorphic iterative centroid in patients with Alzheimer's disease
}

\author{
Claire Cury ${ }^{\mathrm{a}, \mathrm{b}, \mathrm{c}, \mathrm{d}, \mathrm{e}, \mathrm{f} *}{ }^{*}$, Joan Glaunès ${ }^{\mathrm{g}}$, Marie Chupina ${ }^{\mathrm{a}, \mathrm{b}, \mathrm{c}, \mathrm{d}, \mathrm{e}, \mathrm{f}}$, and Olivier Colliot , $^{\mathrm{a}, \mathrm{b}, \mathrm{c}, \mathrm{d}, \mathrm{e}, \mathrm{f}}$ \\ ${ }^{a}$ Inserm, U1127, ICM, F-75013 Paris, France \\ ${ }^{b}$ CNRS, UMR 7225, ICM, F-75013 Paris, France \\ ${ }^{c}$ Sorbonne Universités, UPMC Univ Paris 6, UMR S 1127, ICM, F-75013 Paris, France \\ ${ }^{d}$ Institut du Cerveau et de la Moëlle épinière, ICM, F-75013 Paris, France \\ ${ }^{e}$ Inria, Aramis project-team, Centre Paris-Rocquencourt, France \\ $f$ Centre d'Acquisition et de Traitement des Images (CATI), Paris and Saclay, France \\ ${ }^{g}$ MAP5, Université Paris Descartes, Sorbonne Paris Cité, France
}

(Received 00 Month 20XX; accepted 00 Month 20XX)

\begin{abstract}
This paper presents a new approach for template-based analysis of anatomical variability in populations, in the framework of Large Deformation Diffeomorphic Metric Mappings and mathematical currents. We propose a fast approach in which the template is computed using an diffeomorphic iterative centroid method. Statistical analysis is then performed on the initial momenta that define the deformations between the centroid and each individual subject. We applied the approach to study the variability of the hippocampus in 134 patients with Alzheimer's disease (AD) and 160 elderly control subjects. We show that this approach can describe the main modes of variability of the two populations and can predict the performance to a memory test in $\mathrm{AD}$ patients.
\end{abstract}

Keywords: Shape analysis; LDDMM; Diffeomorphic Iterative Centroid; hippocampus; Alzheimer

\section{Introduction}

Computational Anatomy aims at developing tools for the quantitative analysis of variability of anatomical structures, and its variation in healthy and pathological cases (Grenander \& Miller 1998). A common approach in Computational Anatomy is template-based analysis, where the objective is to compare anatomical objects variations with respect to a common template. These variations are analysed using the ambient space deformations that match each individual structure to the template. The Large Deformation Diffeomorphic Metric Mapping (LDDMM) framework (Beg et al. 2005) has been widely used for the study of the geometric variation of human anatomy. This framework generates metrics between deformable shapes and provides smooth and non ambiguous matchings between objects. Statistical shape analysis is widely used to study the anatomical variability of a population (Vaillant et al. 2004; Durrleman et al. 2008), or to study the correlations between anatomical structures and cognitive or genetic parameters, and this framework also provides interpretable statistics on a population.

Estimating a template from the population in the LDDMM framework, to be used for further template-based statistical analysis, is a computationally expensive task. Several methods have been proposed. Vaillant et al. (2004) proposed a method based on geodesic shooting which iteratively updates a shape by shooting towards the mean directions. The method proposed by Glaunès \& Joshi

*Corresponding author. Email: claire.cury.pro@gmail.com 
(2006) starts from the whole population and estimates a template by co-registering all subjects using a backward scheme. A different approach was proposed in Durrleman et al. (2008, 2012). The method initializes the template with a standard shape, and uses a forward scheme: deformations are defined from the template to the subjects. The method presented by Ma et al. (2008) uses an hyper template which is an extra fixed shape, and optimizes at the same time deformations from the hyper template to the template and deformations from the template to subjects of the population. All these methods are expensive in terms of computation time, due to the number of iterations needed for the convergence of the method.

In this paper, we propose a fast approach for template-based statistical analysis of anatomical variability in the LDDMM framework. The template is estimated using an iterative approach which quickly provides a centroid of the population (Cury et al. 2013, 2014b). This method iteratively computes a centroid of the population in the LDDMM framework, which requires only $N-1$ matching steps, with $N$ the number of subjects, while template estimation methods typically require $N$ such steps per iteration. Then the deformations from the centroid to the subjects of the population are analysed using principal component analysis (PCA), and the resulting shape parameters are used to predict clinical variables using multiple linear regression. The approach was applied to analyse the shape of the hippocampus from a dataset of 134 patients with Alzheimer's Disease (AD) and 160 controls. All the subjects are from the Alzheimer's Disease Neuroimaging Initiative (ADNI) database. Hippocampi were segmented from T1-weighted Magnetic Resonance Images (MRI) and then converted to 3D meshes. This paper extends work that was presented at the MIUA 2014 conference (Cury et al. 2014a). It includes a more detailed description of mathematical approaches as well as new experiments, on a larger dataset, that assess the ability of the method to describe group variability and to predict clinical variables.

\section{Methods}

\section{$2.1 \quad L D D M M$ framework}

We briefly present the LDDMM framework to introduce notations, for more details see Beg et al. (2005). Deformation maps $\varphi: \mathbb{R}^{3} \rightarrow \mathbb{R}^{3}$ are generated via integration of time-dependent vector fields $v(x, t), x \in \mathbb{R}^{3}, t \in[0,1]$, such that each $v(\cdot, t)$ belongs to a Reproducing Kernel Hilbert Space $V$ with kernel $K_{V}$. The transport equation

$$
\begin{cases}\frac{d \phi_{v}}{d t}(x, t)=v\left(\phi_{v}(x, t), t\right) & \forall t \in[0,1] \\ \phi_{v}(x, 0)=x & \forall x \in \mathbb{R}^{3}\end{cases}
$$

has a unique solution, and one sets $\varphi_{v}=\phi_{v}(\cdot, 1)$ the diffeomorphism induced by $v(x, t)$. In a discrete setting, optimal vector fields $v(x, t)$ are expressed as combinations of spline fields: $v(x, t)=\sum_{p=1}^{n} K_{V}\left(x, x_{p}(t)\right) \alpha_{p}(t)$, where $x_{p}(t)=\phi_{v}\left(x_{p}, t\right)$ are the trajectories of control points $x_{p}$ (the vertices of the mesh to be deformed), and $\alpha_{p}(t) \in \mathbb{R}^{3}$ are time-dependent vectors called momentum vectors. Optimal trajectories between shapes can be shown to satisfy geodesic equations for a metric on the set of control points (Vaillant et al. 2004). As a result the full deformation between a template shape and its surface target $S_{i}$ is encoded by the vector of initial momentum vectors $\boldsymbol{\alpha}^{i}(0)=\left(\alpha_{p}^{i}(0)\right)_{1 \leq p \leq n_{i}}$ located on the vertices of the template mesh (or at an initial position $x_{i}(0)$. As a consequence, these initial momentum vectors encode all information of the optimal diffeomorphism between two surfaces. This is a very important point, specifically for group studies, since it allows to analyse the set of deformation maps from a given template to the observed shapes by performing statistics on the initial momentum vectors located on the template shape. We also can use geodesic shooting from initial conditions $\left(x_{i}(0), \alpha_{i}(0)\right)$ in order to generate any arbitrary deformation of a shape in the space of shapes. 


\subsection{Currents framework}

Shapes are represented using the framework of currents introduced in Computational Anatomy by Vaillant \& Glaunès (2005) and Glaunès (2005) and subsequently developed by Durrleman (2010). The idea of currents is to characterize a shape by the collection of the real numbers given in equation 2 for all possible vector fields $w$. Currents provide a dissimilarity measure between meshes which does not assume point-to-point correspondence between anatomical structures. The approach proposed by Vaillant \& Glaunès (2005) is to represent meshes as objects in a linear space and supply it with a computable norm. Using currents to describe surfaces has some benefits. First it avoids the point correspondence issue : one does not need to define pairs of corresponding points between two surfaces to evaluate their spatial proximity. Moreover, metrics on currents are robust to different samplings (i.e. meshes, since surfaces are discretized to be represented via meshes) and topologies and take into account not only the global shapes but also their local orientations. Another important benefit is that the space of currents is a vector space, which allows to consider linear combinations such as means of shapes in the space of currents. Any smooth vector field $w$ of $\mathbb{R}^{3}$ can be integrated over a surface $S$ via the rule:

$$
[S](w)=\int_{S}\langle w(x), n(x)\rangle d \sigma_{S}(x)
$$

with $n(x)$ the unit normal vector to the surface, $d \sigma_{S}$ the Lebesgue measure on the surface $S$. $[S]$ is called a 2-current associated to $S$.

We define a Hilbert metric $\langle\cdot, \cdot\rangle_{W}$ on the space of vector fields of $\mathbb{R}^{3}$ and require that the Hilbert space $W$ to be smooth enough (i.e. continuously embedded in $C_{0}^{1}\left(\mathbb{R}^{3}, \mathbb{R}^{3}\right)$ ). $W^{*}$ is the dual space i.e. the space of continuous linear forms on $W$ to $\mathbb{R}^{3}$. The Riesz representation theorem, states that there exists a unique $u \in W$ such that for all $w \in W,\langle u, w\rangle_{W}=\delta_{x}^{\alpha}(w)=\langle w(x), \alpha\rangle \cdot u$ is thus a vector field which depends on $x$ and linearly on $\alpha$, and we write it $u=K_{W}(\cdot, x) \alpha . K_{W}(x, y)$ is a $3 \times 3$ matrix, and $K_{W}: \mathbb{R}^{3} \times \mathbb{R}^{3} \rightarrow \mathbb{R}^{3 \times 3}$ the mapping called the reproducing kernel of the space $W$. Thus we have the rule $\left\langle K_{W}(\cdot, x) \alpha, w\right\rangle_{W}=\langle w(x), \alpha\rangle$.Applying this formula to $w=K_{W}(\cdot, y) \beta$ for any other point $y \in \mathbb{R}^{3}$ and vector $\beta \in \mathbb{R}^{3}$, we get $\left\langle K_{W}(\cdot, x) \alpha, K_{W}(\cdot, y) \beta\right\rangle_{W}=\alpha^{T} K_{W}(x, y) \beta=\left\langle\delta_{x}^{\alpha}, \delta_{y}^{\beta}\right\rangle_{W^{*}}$ with $\delta_{x}^{\alpha}: w \mapsto\langle w(x), \alpha\rangle \in W^{*}$

One can then prove that for two surfaces $S$ and $T$ :

$$
\langle[S],[T]\rangle_{W^{*}}=\int_{S} \int_{T}\left\langle n_{S}(x), K_{W}(x, y) n_{T}(y)\right\rangle d \sigma_{S}(x) d \sigma_{T}(y) .
$$

We can now define, the optimal matching between two currents $[S]$ and $[T]$, which is the diffeomorphism minimizing the functional

$$
J_{S, T}(v)=\gamma E(v)+\left\|\left[\varphi_{v}(S)\right]-[T]\right\|_{W^{*}}^{2}
$$

with $\mathrm{E}(\mathrm{v})=\int_{0}^{1}\|v(\cdot, t)\|_{V}^{2} d t$ the regularisation term and $\gamma$ the weighting between the two terms, in practice for the Iterative Centroid method we used very small values for gamma. This functional is non convex and in practice we use a gradient descent algorithm to perform the optimization.

\section{$2.3 \quad$ Iterative Centroid method}

Now we have the possibility to match a surface onto an other one to determine the deformation needed to bring the first one onto the other one. We then build a template which is representative of the population, and that will be the cornerstone of the subsequent statistical analysis. Here the template is computed via a diffeomorphic Iterative Centroid method which we previously introduced (Cury et al. 2014b). We proposed three different schemes for centroid computation, but here 
we only use the first one, which is the faster one. The method computes a centroid between two surfaces $S_{1}$ and $S_{2}$ by transporting a first surface $S_{1}$ along the geodesic flow computed by matching (see equation 4) this surface to $S_{2}$. The transport is stopped depending on the iteration number. At the beginning, we have $C_{1}=S_{1}$, so the initial centroid $C_{1}$, represents one surface. To add $S_{2}$ to the centroid, we have to match $C 1$ on $S_{2}$ : the transport is stopped at time $1 /(1+1)$. $C_{2}$ is the result of this transport and is the centroid of 2 surfaces $\left(S_{1}\right.$ and $\left.S_{2}\right)$. We iterate the process by transporting $C_{2}$ along the geodesic going to $S_{3}$, so we stopped the transport at time $1 /(2+1)$ to obtain $C_{3}$. The Iterated Centroid method consists in applying the following procedure (Algorithm 1).

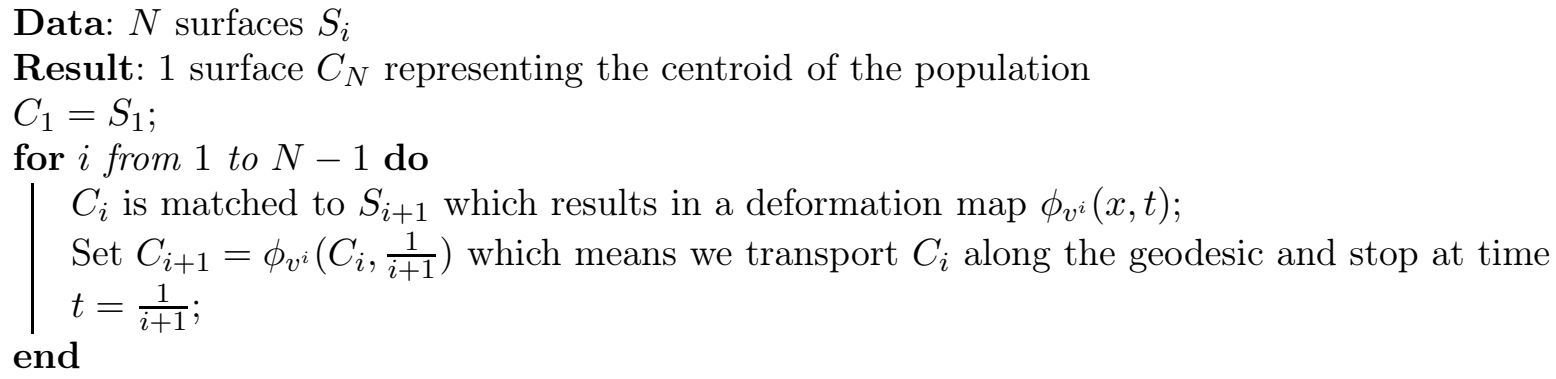

Algorithm 1: Iterative Centroid algorithm

This algorithm can be defined in a pure Riemannian setting, for the averaging of a set of points $p_{k}$ (instead of shapes $S_{i}$ ) on a Riemannian manifold $M$. If points $p_{k}$ belong to a vector space and the metric is flat, the algorithm converges towards the mean $\frac{1}{N} \sum_{k} p_{k}$, but in general it depends on the ordering of the points. Back to our shape space framework, we showed in a previous study (Cury et al. 2014b, 2013) that indeed the ordering changes the final centroid, but quantitative evaluations show that the different results are very close to each other. Emery \& Mokobodzki (1991) proposed to define the centroid not as a unique point but as the set $\mathcal{C}_{N}$ of points $p \in M$ satisfying $f(p) \leq$ $\frac{1}{N} \sum_{k=1}^{N} f\left(p_{k}\right)$, for any convex function $f$ on $M$ (a convex function $f$ on $M$ being defined by the property that its restriction to all geodesics is convex). This set $\mathcal{C}_{N}$ takes into account all centroids obtained by bringing together points $p_{k}$ by all possible means, i.e. recursively by pairs, or by iteratively adding a new point, as we are doing with Algorithm 1.

For both kernels, the kernel of the space of deformation $K_{V}$ and the kernel of the space of currents $K_{W}$, we used Cauchy kernels i.e. kernels of the form $K_{W}(x, y)=h\left(\|x-y\|^{2} / \sigma_{W}^{2}\right) I_{3}$, where $h$ is the real function $h(r)=1 /(1+r)$. The parameters, which main ones are the sizes of both kernels have to be adapted to the size of the data: the kernel $K_{V}$ has to be enough large to capture all subjects of the population, here we used for these data $\sigma_{V}=13$, and the kernel $K_{W}$ has to be smaller to capture local differences between shapes, the optimal match between two surfaces are computed using a multi scale approach using decreasing values of $\sigma_{W}$, here the smallest value was $\sigma_{W}=3$. To further reduce the computational load, we used a GPU implementation for the kernel convolutions involved in the matchings.

\section{$2.4 \quad$ Principal Component Analysis}

For the statistical analysis of shapes, we compute a centroid of the population, and we use it as a template. Then we deform the centroid toward each shape of the population to obtain the deformations from the centroid to the population. The deformations are determined by the vector of initial momentum vectors $\boldsymbol{\alpha}^{i}(0)$. We can analyse these deformations with a Principal Component Analysis (PCA) on the initial momentum vectors as in Vaillant et al. (2004). However, unlike in standard PCA, the covariance matrix computation needs to include the kernel $K_{V}$ of the space of deformation $V$ :

$$
\operatorname{Cov}(i, j)=\frac{1}{N-1}\left(\boldsymbol{\alpha}^{i}(0)-\overline{\boldsymbol{\alpha}}(0)\right) K_{V}(x)\left(\boldsymbol{\alpha}^{j}(0)-\overline{\boldsymbol{\alpha}}(0)\right)
$$


with $\overline{\boldsymbol{\alpha}}(0)$ the mean of initial momentum vectors and $K_{V}(x)$ the matrix of the $K_{V}\left(\boldsymbol{x}^{i}, \boldsymbol{x}^{j}\right)$. The $k$-th principal mode is computed from the $k$-th eigenvector $\nu^{k}$ of $C_{V}$, as follows:

$$
\boldsymbol{m}^{k}=\overline{\boldsymbol{\alpha}}+\sum_{j=1}^{N} \nu_{j}^{k}\left(\boldsymbol{\alpha}^{j}-\overline{\boldsymbol{\alpha}}\right) .
$$

The cumulative explained variance $C E V_{k}$ for the $k$ first principal modes is given by equation:

$$
C E V_{k}=\frac{\sum_{h=1}^{k} \lambda_{h}}{\sum_{h=1}^{N} \lambda_{h}},
$$

with $\lambda_{h}$ the $h$-th largest eigenvalue corresponding to the $h$-th principal eigenvector $\nu^{h}$. We can use geodesic shootings along any principal mode $\boldsymbol{m}^{k}$ to visualise the corresponding deformations.

\subsection{Multiple linear regression}

From the space computed via the PCA, by using the z-scored $p$ principal eigenvectors $X_{1, i}, \ldots, X_{p, i}$ with $i \in\{1, \ldots, N\}$ of the $N$ subjects, we can use multiple linear regression to predict a biological or clinical factor $Y$. The multiple linear regression model is written as $f(X)=\beta_{0}+\sum_{i=1}^{p} X_{i} \beta_{i}$ where $\beta_{0}, \beta_{1}, \ldots \beta_{p}$ are the regression coefficients. The standard method to estimate the regression coefficients is the least squares estimation method in which the coefficients $\beta_{i}$ minimize the residual sum of squares $R S S(\beta)=\sum_{j=1}^{N}\left(y_{j}-\beta_{0}-\sum_{i=1}^{p} x_{j i} \beta_{i}\right)^{2}$, which leads to the estimated $\hat{\beta}$ (with matrix notations) $\hat{\beta}=\left(X^{T} X\right)^{-1} X^{T} Y$ (Hastie et al. 2009). For each dimension $p$ we validated the quality of the computed model with the adjusted coefficient of determination $R_{a d j}^{2}$, which expresses the part of explained variance of the model with respect to the total variance:

$$
R_{a d j}^{2}=1-\frac{S S E /(N-p)}{S S T /(N-1)}
$$

with $S S E=\sum_{i}^{N}\left(y_{i}-\left(X_{1 \ldots p, i}^{T} \hat{\beta}\right)\right)^{2}$ and $S S T=\sum_{i=1}^{N}\left(y_{i}-\bar{Y}\right)^{2}$. The $R_{a d j}^{2}$ coefficient, unlike the $R^{2}$, takes into account the number of variables and therefore does not increase with the number of variables. We then tested the significance of each model by computing the F statistic

$$
F=\frac{R^{2} / p}{\left(1-R^{2}\right) /(N-p-1)}
$$

which follows a F-distribution with $(p, n-p-1)$ degrees of freedom. So for each number of variables (i.e. dimensions) we computed the coefficient of determination to evaluate the model and the $p$ value to evaluate the significance of the model. Then we used a leave-one-out cross validation which consists in computing $N$ models with $N-1$ observations to predict the remaining one. To quantify the prediction of the model, we computed the mean square error $M S E=S S E / N$ which corresponds to the unexplained residual variance.

\section{Experiments and results}

\subsection{Dataset and experiments}

The method was applied to the analysis of hippocampal shapes of 134 patients with Alzheimer's Disease $(\mathrm{AD}$, with age $=75.8 \pm 7.3$ years, $50 \%$ male, $\mathrm{MMSE}=23$ in average between 18 and 27$)$ and 
160 controls $(\mathrm{CN}$, with age $=76.0 \pm 5.4$ years, $47 \%$ male, MMSE $=29$ in average, between 25 and 30) $\left(N=294\right.$ in total) from the Alzheimer's Disease Neuroimaging Initiative (ADNI) database ${ }^{1}$. Left hippocampi were automatically segmented with the SACHA software (Chupin et al. 2009) from 3D T1 weighted MRI. Briefly, this method is fully automatic and is based on competitive region growing with anatomical constraints. Then the meshes were computed using a marching cube method implemented in the BrainVisa software (http://www.brainvisa.info). Hippocampal meshes are composed of 800 vertices on average. Examples of meshes with 686 and 1059 vertices are shown on Figure 1.

For this dataset, we first analysed the variability of both patients and control groups using Principal Component Analysis. Then, for the AD group, we assessed the aptitude of our approach to predict clinical parameters. Specifically, we studied the Mini Mental State Examination (MMSE) which is a global indicator of the severity of cognitive impairment, and the ADNI-MEM score (Crane et al. 2012) which is composite score reflecting the performance of the subject on MEMory tasks. The average ADNI-MEM score for AD group is -0.88 and for the CN group is 0.98 .

We hypothesise that hippocampal shape predicts the ADNI-MEM score, reflecting the central role of the hippocampus in memory performance. On the other hand, we hypothesise that it will not predict the MMSE which is a
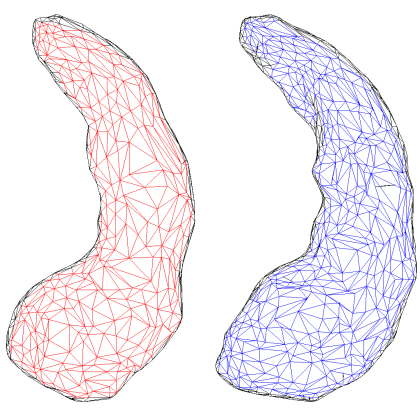

Figure 1. Examples of hippocampal meshes computed from an AD patient (left) and from a CN subject (right). the left mesh is composed by 686 vertices and the right is composed by 1059 vertices.

\subsection{Results: analysis of variability}

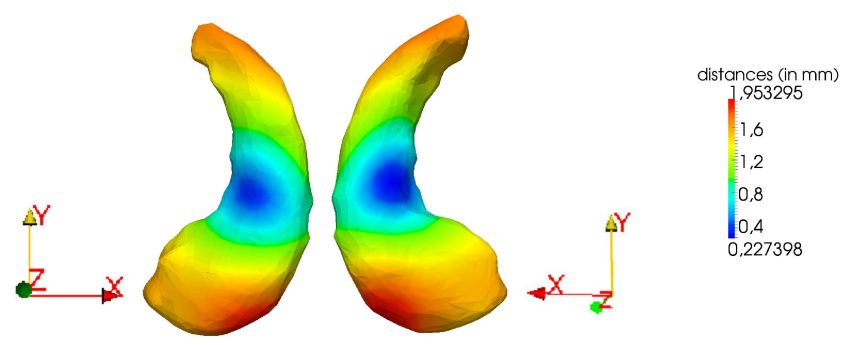

Figure 2. Distances between $I C(C N)$ and the deformation of $I C(C N)$ onto $I C(A D)$. On the left, the hippocampus is viewed from below.

We computed a centroid for each of the two populations (AD and $\mathrm{CN}$ ) using Algorithm 1. The two centroids are denoted $I C(A D)$ and $I C(C N)$. Computation times were 2.4 hours for $I C(A D)$ and 3.6 hours for $I C(C N)$. To assess whether the centroids are close to the center of the respective populations, we computed the ratio $R=\frac{\left\|\frac{1}{N} \sum_{i=1}^{N} v_{0}\left(S_{i}\right)\right\|_{V}}{\frac{1}{N} \sum_{i=1}^{N}\left\|v_{0}\left(S_{i}\right)\right\|_{V}}$ between the mean of the norms of initial vector fields from the centroid to the population and the norm of the mean of initial vector fields. Both ratios are 0.25 , which means that both centroids are correctly centred even though they are not exactly at the Fréchet mean (which would correspond to $R=0$ ). To visualise differences between

\footnotetext{
${ }^{1}$ (adni.loni.usc.edu). The Principal Investigator of this initiative is Michael W. Weiner, MD, VA Medical Center and University of California - San Francisco. The investigators within the ADNI contributed to the design and implementation of ADNI and/or provided data but did not participate in analysis or writing of this report A complete listing of ADNI investigators can be found at: adni.loni.usc.edu/wp-content/uploads/how to_apply/ADNI_Acknowledgement_List.pdf. Data collection and sharing for this project was funded by the Alzheimer's Disease Neuroimaging Initiative (ADNI) (National Institutes of Health Grant U01 AG024904) and DOD ADNI (Department of Defense award number W81XWH-12-2-0012).
} 

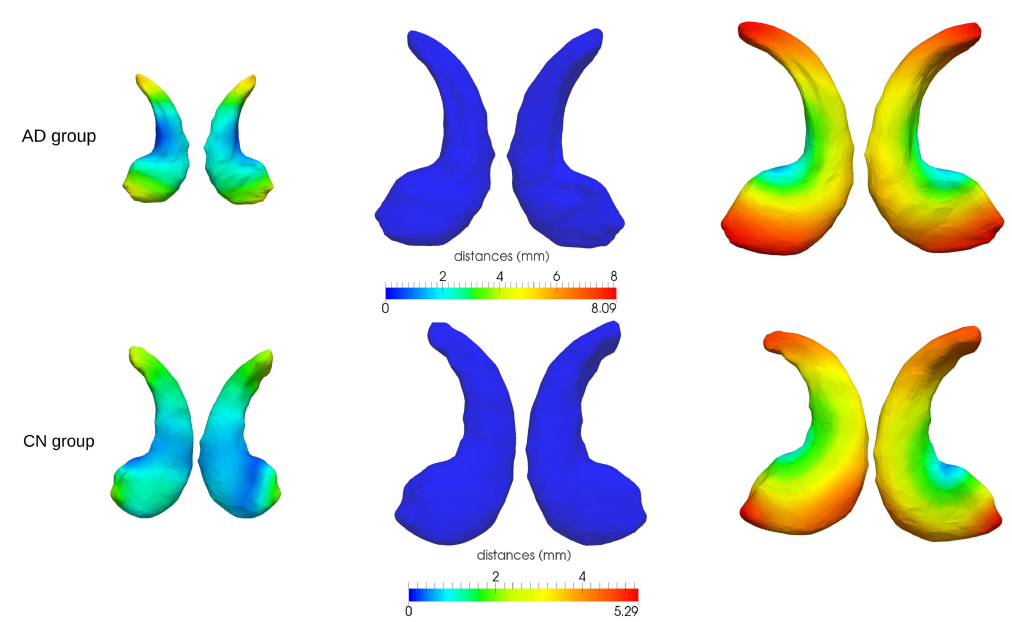

Figure 3. First mode of deformation of the AD group (top) and of the CN group (bottom). For each row, the centroid is in the center (in blue), on the right its deformation at $+2 \sigma_{1} \boldsymbol{m}^{1}$, and at $-2 \sigma_{1} \boldsymbol{m}^{1}$ on the left. The colormap indicates the displacement of each vertex between the corresponding centroid and its deformation.

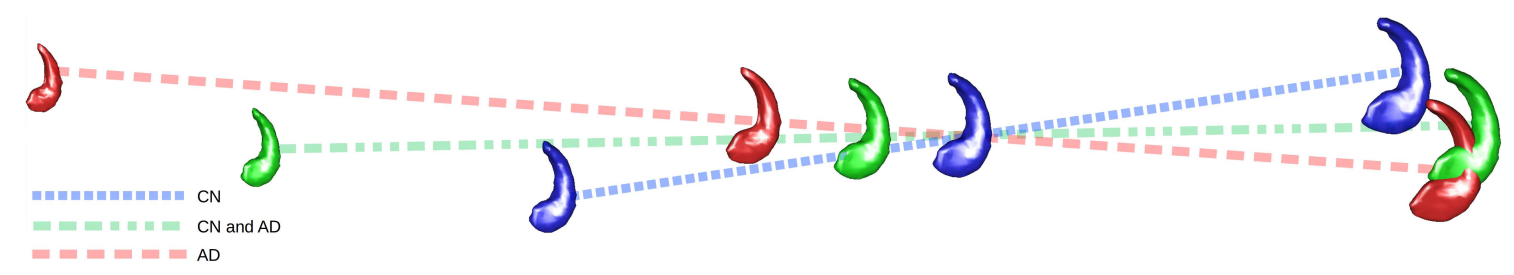

Figure 4. Two principal axes of the whole population (in green), the length of each axis is proportional to its standard deviation. In blue, the projections of $I C(C N)$ and projections of its deformations at $\pm 2 \sigma$ in the direction of its first mode of variation. In red, for the $\mathrm{AD}$ group.

$I C(C N)$ and $I C(A D)$, we computed distances between vertices of $I C(C N)$ and the deformation of $I C(C N)$ onto $I C(A D)$ (figure 2).

We then analysed the variability of the $\mathrm{AD}$ and the $\mathrm{CN}$ populations using PCA. Figure 3 shows, for each group, the principal mode of variation. This figure is obtained by geodesic shooting from each centroid in the first principal direction with a magnitude of $\pm 2 \sigma_{1}$, with $\sigma_{1}$ the standard deviation of the first mode of variation. One can note that, while the templates of the two groups are different, the variabilities of both groups share similarities. Nevertheless, there seems to be less variability in the medial part of the body for the CN group.

In order to visualize the localization of $I C(C N)$ and $I C(A D)$ and the corresponding first modes within the whole population, we computed a centroid of the whole population and performed a PCA. We then projected $I C(C N)$ and $I C(A D)$ and their corresponding $\pm 2 \sigma_{1} \boldsymbol{m}^{1}$ onto the $2 \mathrm{D}$ space spanned by the first two principal components of the whole dataset. We can observe (figure 4) that $I C(C N)$ is on the right of the global centroid, and $I C(A D)$ is on the other side, and the 3 principal modes of variation have different directions. One can observe that for the AD and the CN group, the two shapes corresponding to the $+2 \sigma_{1} \boldsymbol{m}^{1}$ deformation (on the right of the figure 4) are close i.e. similar to each others. This observation can also be note on the figure 3 , which shows that the deformation in the direction $-2 \sigma_{1}$ for the $\mathrm{AD}$ group is very different than the one of the CN group and the deformation in the direction $+2 \sigma_{1}$ for the $\mathrm{AD}$ group is similar to the one of the $\mathrm{CN}$ group.

We then studied the variance associated to the different dimensions. In total, the AD group have more variance than the CN group: $\sum_{i=1}^{N_{A D}} \lambda_{i}^{A D}=233$ and $\sum_{i=1}^{N_{C N}} \lambda_{i}^{C N}=171$. The first two components of the $\mathrm{AD}$ group have a variance of respectively 51.4 and 21.2 , while the two first components of the $\mathrm{CN}$ group have a smaller variance of respectively 19.0 and 8.3. We also studied the proportion of cumulative variance $C E V_{k}$ explained for each number of dimensions $k$. On Figure 5, we can see that the AD group needs less dimensions (or principal components), around 40 dimensions, 


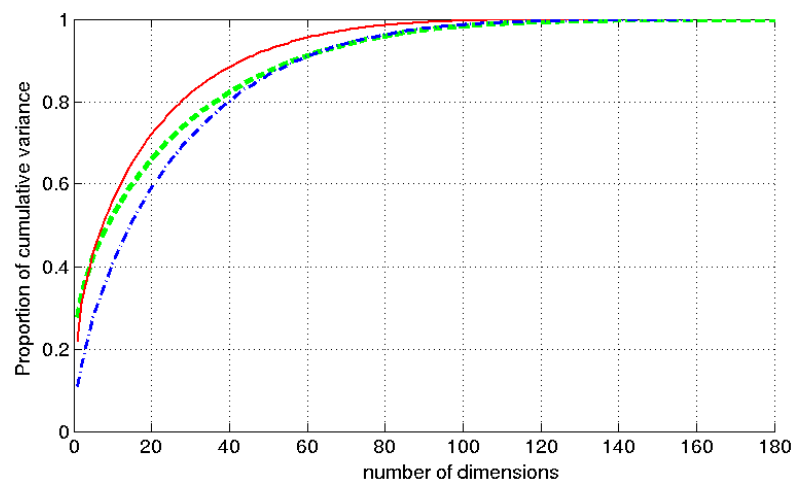

Figure 5. Results of proportion of cumulative variance explained regarding to the number of dimension computed by PCA. Red line for the AD group, '-.-' blue line for the CN group and dotted green line for the whole population.

to explain $90 \%$ of its total variance than the $\mathrm{CN}$ group (requiring around 60 dimensions). To make sure that this difference is not due to the larger number of subjects in the CN group, we also performed the same experiment with the same number of subjects (134) in each group, and obtained similar results.

Figure 6 shows these two first modes of variations in blue for the CN group and in red for the AD group computed by geodesic shooting in the directions $\left(k_{1} \times \boldsymbol{m}^{1} ; k_{2} \times \boldsymbol{m}^{2}\right)$ with $k_{1} \in\left[-2 \sigma_{1} ;+2 \sigma_{1}\right]$ and $k_{2} \in\left[-2 \sigma_{2} ;+2 \sigma_{2}\right]$,with $\boldsymbol{m}^{1}$ and $\boldsymbol{m}^{2}$ the two first modes of variations of the population, and $\sigma_{i}$ the standard deviation of the corresponding mode $\boldsymbol{m}^{i}$. We can see that for the two groups, the first mode mainly captures the volume effect which effect is clearly (and expected to be) more important for the AD group, and also captures the curvature of the hippocampus tail. The second axis of the $\mathrm{AD}$ group captures the atrophy of the hippocampus body and head.

\subsection{Results: prediction of clinical variables}

For the AD group we tested the ability of different models computed using different number of variables to predict clinical variables (MMSE and ADNI-MEM). The variables of the models are the principal modes of variation i.e. principal components of the PCA computed from the PCA. The response $Y$ is the response to the ADNI-MEM test or to the MMSE test. We saw in the previous section that the $\mathrm{AD}$ group can express $90 \%$ of its total variability by using only 40 dimensions, thus we studied models with maximum 50 principal modes of variation.

Figure 7 shows, for the hippocampi of the $\mathrm{AD}$ group, the values of $R_{a d j}^{2}$ coefficient computed from the models computed with the $k$ first modes of variations, then the corresponding $p$-values are displayed. We cross validated the models with the best $R_{a d j}^{2}$ coefficients and the best $p$-values with the lower number of dimensions. Thus, for the cross validation step we assessed models from 29 dimensions to 40 dimensions which seems to be the more interesting. For these model, $R_{a d j}^{2}=0.25$ on average, and the $p$-values are $1.2 \mathrm{e}-3$ on average. Results of cross validation using leave-one-out (LOOCV) are assessed using the MSE score (right panel of the Figure 7). MSE scores of the predicted values from the cross validation step are compared to the $M S E$ of the model, and to a random prediction distributed normally with mean $\bar{Y}$ and variance $\sigma_{Y}^{2}$.

None of the models (even those computed using more than 50 variables) was able to predict the response to the MMSE test, the maximum value for the $R_{a d j}^{2}$ coefficient was 0.11 , and the $p$-values were all higher than 0.1 .

\section{Conclusion}

In this paper, we proposed a fast template-based shape analysis approach using the LDDMM framework. We estimated the template using a diffeomorphic iterative centroid method. The Iterative 


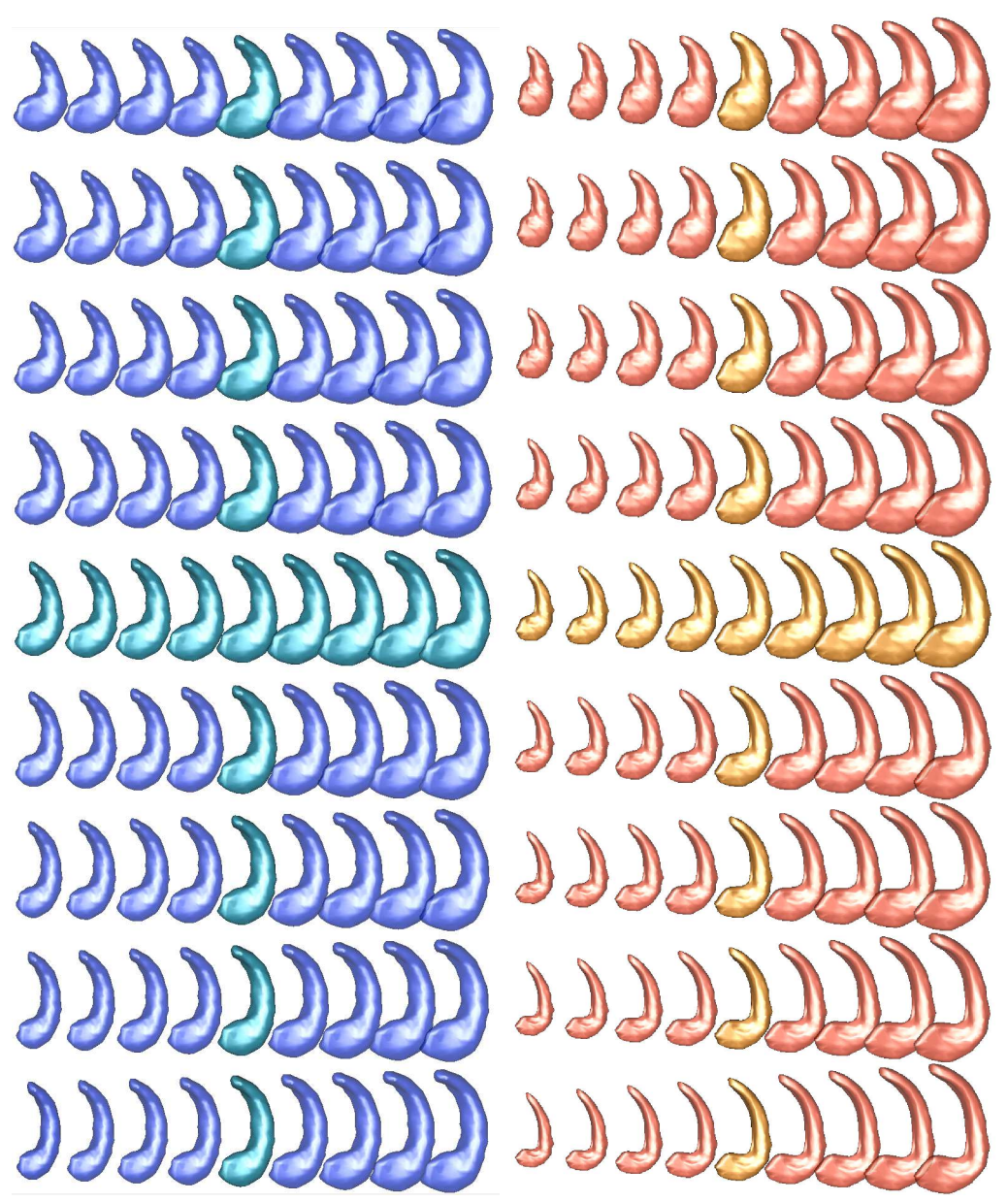

Figure 6. Geodesic shootings in the directions of $\left(k_{1} \times \boldsymbol{m}^{1} ; k_{2} \times \boldsymbol{m}^{2}\right)$ with $k_{1} \in\left[-2 \sigma_{1} ;+2 \sigma_{1}\right]$ and $k_{2} \in\left[-2 \sigma_{2} ;+2 \sigma_{2}\right]$ with $\boldsymbol{m}^{1}$ and $\boldsymbol{m}^{2}$ the two first modes of variations for the AD group (in red) and the CN group (in blues). Principal axis $\left(k_{1} \times \boldsymbol{m}^{1} ; 0\right)$ and $\left(0 ; k_{2} \times \boldsymbol{m}^{2}\right)$ are in yellow for the $\mathrm{AD}$ group and in light blue for the $\mathrm{CN}$ group.

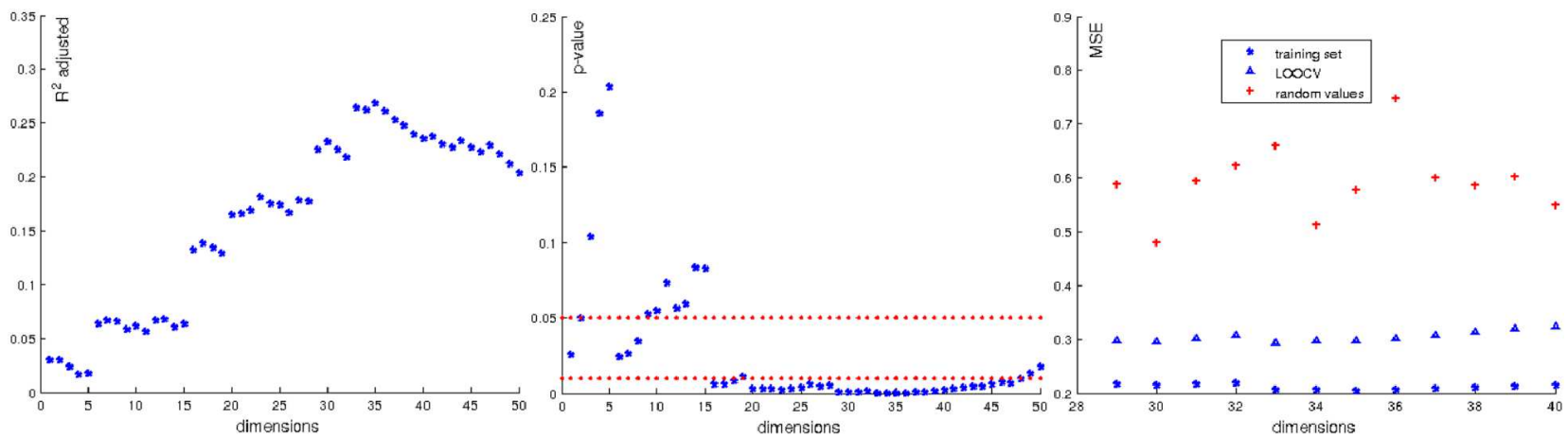

Figure 7. Multiple linear regression on principal components for the $Y$ response ADNI MEM, and variables are the principal modes of variations. On left panels, $R_{a d j}^{2}$ coefficients regarding to the number of dimensions (i.e. viariables) of the model. On middle panels, the corresponding $p$-values of the models with dotted red lines representing the thresholds 0.05 and 0.01 . On right panels, the different $M S E$ computed from some model previously selected using the two previous panels in which the corresponding models are highlighted in green.

Centroid is roughly centred within the population of shapes. Analysis of variability was then based on a PCA on the initial momentum vectors from the template to the subjects. In a future work we will study the impact of different centroids of a population on the subsequent PCA. Projection of the templates of the $\mathrm{CN}$ group and the $\mathrm{AD}$ group onto the main modes of variability of the whole population show that they are located on the main axis of variability. The analysis of the number of dimensions given by the PCA shows that, even if the AD group is more variable than the CN group, 
the AD group is explained by fewer dimensions than the CN group. Our experiments also showed that the approach can be used to predict clinical variables from hippocampal shape. Specifically, we were able to predict memory performances in $\mathrm{AD}$ patients, which is consistent with the central role played by the hippocampus in memory processes.

\section{Acknowledgements}

The research leading to these results has received funding from ANR (project HM-TC, grant number ANR-09-EMER-006, and project KaraMetria, grant number ANR-09-BLAN-0332), from the CATI Project (Fondation Plan Alzheimer) and from the program "Investissements d'avenir" ANR-10IAIHU-06.

\section{References}

Beg, M. F., Miller, M. I., Trouvé, A., \& Younes, L. (2005). Computing large deformation metric mappings via geodesic flows of diffeomorphisms. International Journal of Computer Vision, 61(2), 139-157.

Chupin, M., Hammers, A., Liu, R. S. N., Colliot, O., Burdett, J., Bardinet, E., Duncan, J. S., Garnero, L., \& Lemieux, L. (2009). Automatic segmentation of the hippocampus and the amygdala driven by hybrid constraints: Method and validation. NeuroImage, 46(3), 749-761.

Crane, P. K., Carle, A., Gibbons, L. E., Insel, P., Mackin, R. S., Gross, A., Jones, R. N., Mukherjee, S., Curtis, S. M., Harvey, D., et al. (2012). Development and assessment of a composite score for memory in the Alzheimer's Disease Neuroimaging Initiative (ADNI). Brain imaging and behavior, 6(4), 502-516.

Cury, C., Glaunès, J. A., Chupin, M., Colliot, O., et al. (2014a). Fast Template-based Shape Analysis using Diffeomorphic Iterative Centroid. In MIUA 2014 - Medical Image Understanding and Analysis 2014 (pp. 39-44).

Cury, C., Glaunès, J. A., \& Colliot, O. (2013). Template Estimation for Large Database: A Diffeomorphic Iterative Centroid Method Using Currents. In F. Nielsen \& F. Barbaresco (Eds.), GSI13 - Geometric Science of Information 2013, volume 8085 of Lecture Notes in Computer Science (pp. 103-111). Springer.

Cury, C., Glaunès, J. A., \& Colliot, O. (2014b). Diffeomorphic Iterative Centroid Methods for Template Estimation on Large Datasets. In Geometric Theory of Information (pp. 273-299). Springer.

Durrleman, S. (2010). Statistical models of currents for measuring the variability of anatomical curves, surfaces and their evolution. PhD thesis, University of Nice-Sophia Antipolis.

Durrleman, S., Pennec, X., Trouvé, A., Ayache, N., et al. (2008). A forward model to build unbiased atlases from curves and surfaces. In 2nd MICCAI Workshop on Mathematical Foundations of Computational Anatomy (pp. 68-79).

Durrleman, S., Prastawa, M., Korenberg, J. R., Joshi, S., Trouvé, A., \& Gerig, G. (2012). Topology Preserving Atlas Construction from Shape Data without Correspondence Using Sparse Parameters. In MICCAI 2012 - Medical Image Computing and Computer-Assisted Intervention, LNCS (pp. 223-230).: Springer.

Emery, M. \& Mokobodzki, G. (1991). Sur le barycentre d'une probabilité dans une variété. In Séminaire de probabilités, volume 1425 of Lecture Notes in Mathematics (pp. 220-233). Springer.

Glaunès, J. A. (2005). Transport par difféomorphismes de points, de mesures et de courants pour la comparaison de formes et l'anatomie numérique. PhD thesis, Université Paris 13.

Glaunès, J. A. \& Joshi, S. (2006). Template estimation from unlabeled point set data and surfaces for Computational Anatomy. In the International Workshop on the MFCA-2006 (pp. 29-39).

Grenander, U. \& Miller, M. I. (1998). Computational anatomy: An emerging discipline. Quarterly of applied mathematics, 56(4), 617-694.

Hastie, T., Tibshirani, R., Friedman, J., Hastie, T., Friedman, J., \& Tibshirani, R. (2009). The elements of statistical learning. Springer.

Ma, J., Miller, M. I., Trouvé, A., \& Younes, L. (2008). Bayesian template estimation in computational anatomy. NeuroImage, 42(1), 252-261.

Vaillant, M. \& Glaunès, J. A. (2005). Surface matching via currents. In Information Processing in Medical Imaging (pp. 381-392).: Springer.

Vaillant, M., Miller, M. I., Younes, L., \& Trouvé, A. (2004). Statistics on diffeomorphisms via tangent space representations. NeuroImage, 23, S161-S169. 